\title{
Research on Mechanical and Electrical Properties of Cu-Ag Alloys Designed for the Construction of High Magnetic Field Generators
}

\author{
Artur Kawecki $^{*} \mathbb{C}$, Eliza Sieja-Smaga $_{\mathbb{C}}$, Kinga Korzeń $_{\mathbb{C}}$, Magdalena Majchrowska $_{\mathbb{C}}$, Piotr Noga $_{\mathbb{C}}$ \\ AGH University of Science and Technology, Faculty of Non-Ferrous Metals, Mickiewicza 30 Ave, 30-059 Krakow, Poland \\ *e-mail: akawecki@agh.edu.pl
}

(C) 2021 Authors. This is an open access publication, which can be used, distributed and reproduced in any medium according to the Creative Commons CC-BY 4.0 License requiring that the original work has been properly cited.

Received: 27 November 2021/Accepted: 20 December 2021/Published online: 29 December 2021.

This article is published with open access at AGH University of Science and Technology Journals.

\begin{abstract}
The individual sections, wiring and construction of electromagnet windings responsible for strong magnetic field impulses may be one application for hypoeutectic $\mathrm{Cu}$-Ag alloys. High electrical properties and mechanical properties (tensile strength, yield strength, impact strength) as well as high heat, fatigue and rheological resistance are required for these kinds of applications due to the unique nature of such operations (strong vibrations of high frequency and amplitude resulting from Lorenz forces and the possibility of significant and rapid heating from Jule's heat). The limited solubility of copper and silver in the solid state enables the effective modification of the alloys' microstructure through heat treatment and further shaping of their high mechanical and electrical properties via cold plastic working. The article presents the manufacturing of Cu-Ag alloys with the weight percent of Ag between 3 and 7 using the continuous casting process along with research on the physicochemical, mechanical and electrical properties of the obtained casts. The research on the amount of plastic deformation and its influence on the wire drawing process and the mechanical and electrical properties of the wires is also discussed. The temperature coefficients of resistance were defined in order to determine the temperature influence on the electrical resistance changes dynamics. The microstructural analysis was carried out in the as-cast state. The preliminary research conducted indicates that the obtained Cu-Ag alloys in the as-cast state exhibit a set of high mechanical and electrical properties. The prospective next stage of research includes the selection of favourable heat treatment parameters which would provide optimally modified microstructure of the alloys, as well as determining the deformation coefficients allowing for further increases in the mechanical and electrical properties.
\end{abstract}

\section{Keywords:}

copper-silver alloys, high magnetic field, continuous casting, wires, high electrical and mechanical properties

\section{INTRODUCTION}

The magnetic field is used, among others, as an experimental research tool in various branches of science e.g. physics of condensed matter, molecular physics, chemistry and increasingly biology. The constant increasing of dimensions, the construction complexity and installation costs of electromagnets needed to achieve the extremely high intensity magnetic field require novel materials with high operational properties. For instance, the windings of electromagnet coils responsible for generating strong magnetic field impulses should be manufactured from materials characterized by high strength, impact and fatigue resistance and high electrical conductivity properties [1-3]. The windings of the electromagnet operate at the cryogenic conditions as a result of causing short term electromagnetic impulses (a fraction of a second), which generate Lorenz forces with very high values directed from the centre of the coil outwards. These values are so significant that they may lead to the explosive destruction of not only the coil windings but also the electromagnet's body. The ideal material for the manufacture of the electromagnet coil winding should combine the high mechanical properties of steel with the electrical conductivity of a superconductor or copper [4-8]. Only then, due to the proper design of the coil and high intensity electric current flowing through the winding, may a magnetic field of a very high value be created. However, to date, such a material does not exist. Nowadays, superconducting materials are more commonly used to construct electromagnets, however, they do not have sufficiently high strength properties, therefore they are usually reinforced with, for instance, composite materials or and steel $[9,10]$. $\mathrm{Cu}$-Ag alloys, due to their specific material properties, may be used to construct the generators of strong magnetic fields [11-14]. The limited solubility of both copper and silver at a solid state as the temperature decreases and the eutectic transformation at $779^{\circ} \mathrm{C}$ with $71.9 \mathrm{wt} . \%$ of eutectic silver content is characteristic of $\mathrm{Cu}-\mathrm{Ag}$ alloys. At the temperature of eutectic transformation, the maximum solubility of silver in copper is equal to $8 \mathrm{wt} . \%$, and copper in silver is equal to 8.8 wt.\%. The mutual solubility of both alloy components decreases as the alloy temperature decreases in agreement with the boundary line of solubility of the alloying elements. 
The appropriate heat treatment (supersaturation and aging) allows for controlled and effective modification of the alloy microstructure through mutual precipitation reactions, which may theoretically lead to almost complete separation of the alloy element from the matrix [15-18]. The shape, size and number of precipitates depend on the alloy additive content and the heat treatment parameters. If such an alloy is subjected to cold plastic working, e.g. in the wire drawing process, the structural effect will be the presence of numerous, highly elongated fibres of nearly pure silver in a matrix of almost pure copper. The silver fibre cross-section may reach nanometric dimensions depending on the parameters of heat treatment and the amount of deformation applied. Thus, a reinforcing composite phase is formed which, due to plastic deformation and generated structural defects, contributes strongly to the strengthening of the alloy. The electrical properties of the alloy increase as the alloy component is removed from the matrix. Therefore, the combination of precipitation hardening and strain hardening of $\mathrm{Cu}-\mathrm{Ag}$ alloys may consequently lead to obtaining a material with very high mechanical and electrical properties [19-21]. Such features of $\mathrm{Cu}-\mathrm{Ag}$ alloys may be used to obtain wires characterized by a set of very high mechanical and the requisite electrical properties required for electrotechnical applications [22].

\section{MATERIALS AND METHODOLOGY}

Hypoeutectic, single-phase alloys (CuAg3; CuAg4; CuAg5; CuAg6; CuAg7) were selected as research materials. Castrods of $9.5 \mathrm{~mm}$ diameter were obtained using a laboratory continuous melting and casting induction furnace consisting of a generator, an electronic control system of the heating and casting process and a cast-rod pulling system. As the constant movement of the liquid metal is possible due to the induction heating of the crucible, the repeatability of the chemical composition is achieved along the whole length of the cast-rods. The crucibles and crystallizers used during the continuous casting process were manufactured of high-purity pressed graphite. In order to provide maximal deoxidization of the alloy, a graphite powder was used to cover the liquid metal in the crucible. A protective nitrogen gas was supplied both inside the crucible and the area of the crystallizer to protect the molten metal against oxygen and provide longer operating time of graphite elements. The continuous casting parameters are presented in Table 1 . The temperatures of the liquid metal in the crucible ranged between $1240^{\circ} \mathrm{C}$ and $1280^{\circ} \mathrm{C}$ during the process and the solidified cast-bars immediately after the crystallizer ranged between $170^{\circ} \mathrm{C}$ and $190^{\circ} \mathrm{C}$. The casting feed during the process was equal to $3 \mathrm{~mm} / \mathrm{s}$ with a standstill of $1 \mathrm{~s}$ needed for the liquid metal to flow into the crystallizer. The velocity values of the cooling medium in the primary system (crystallization system) and secondary system (direct cooling of the cast-rod after leaving the crystallizer) as well as the temperature of the cooling medium before entering the crystallization system (T1) and after leaving it (T2) were also presented. The alloying elements were oxygen-free copper (OFC) and granulated silver of $99.99 \%$ chemical purity. The obtained cast-rods were tested in terms of their physicochemical, mechanical and electrical properties. Research on the influence of the amount of plastic deformation throughout the drawing process of cast-rods and the shaping of their mechanical and electrical properties was carried out. Microstructural analyses of the obtained $\mathrm{Cu}-\mathrm{Ag}$ alloys were also conducted in the as-cast state

Table 1

The continuous casting parameters of $\mathrm{Cu}-\mathrm{Ag}$ cast-rods

\begin{tabular}{|c|c|c|c|c|}
\hline Material & $\begin{array}{c}\text { Liquid } \\
\text { metal tem- } \\
\text { perature }\end{array}$ & $\begin{array}{l}\text { Metal tem- } \\
\text { perature } \\
\text { after the } \\
\text { crystallizer }\end{array}$ & Feed & Standstill \\
\hline & \multicolumn{2}{|c|}{$\left[{ }^{\circ} \mathrm{C}\right]$} & {$[\mathrm{mm} / \mathrm{s}]$} & [s] \\
\hline CuAg3 & $1240-1250$ & $170-180$ & 3 & 1 \\
\hline $\mathrm{CuAg} 4$ & $1240-1250$ & $170-180$ & 3 & 1 \\
\hline CuAg5 & $1250-1260$ & $180-190$ & 3 & 1 \\
\hline CuAg6 & $1250-1270$ & $170-180$ & 3 & 1 \\
\hline CuAg7 & $1260-1280$ & $180-190$ & 3 & 1 \\
\hline \multirow[t]{2}{*}{ Material } & $\begin{array}{l}\text { Primary } \\
\text { cooling } \\
\text { velocity }\end{array}$ & $\begin{array}{l}\text { Secondary } \\
\text { cooling } \\
\text { velocity }\end{array}$ & $\begin{array}{l}\text { Water } \\
\text { temp. T1 }\end{array}$ & $\begin{array}{c}\text { Water } \\
\text { temp. T2 }\end{array}$ \\
\hline & \multicolumn{2}{|c|}{$[1 / \mathrm{min}]$} & \multicolumn{2}{|c|}{$\left[{ }^{\circ} \mathrm{C}\right]$} \\
\hline CuAg3 & 0.75 & 0.27 & 20.5 & 37 \\
\hline CuAg4 & 0.75 & 0.35 & 20.7 & 37 \\
\hline CuAg5 & 0.80 & 0.31 & 20.5 & 36 \\
\hline CuAg6 & 0.80 & 0.35 & 20.6 & 38 \\
\hline CuAg7 & 0.85 & 0.38 & 20.8 & 39 \\
\hline
\end{tabular}

\section{RESULTS AND DISCUSSION}

The chemical compositions of the obtained alloys are presented in Table 2. As can be seen, the chemical composition analyses proved the amount of impurities to be insignificant and the oxygen content did not exceed $12 \mathrm{ppm}$. The actual silver content in the alloys was close to the assumed nominal value.

Table 2

Chemical composition of the obtained alloys

\begin{tabular}{cccccc}
\hline \multirow{2}{*}{ Element } & CuAg3 & CuAg4 & CuAg5 & CuAg6 & CuAg7 \\
\cline { 2 - 6 } & \multicolumn{5}{c}{ Amount of impurities [ppm] } \\
\hline $\mathrm{As}$ & 0.57 & 0.49 & 0.77 & 0.83 & 0.65 \\
\hline $\mathrm{Bi}$ & 0.08 & 0.18 & 0.16 & 0.11 & 0.34 \\
\hline $\mathrm{Se}$ & 0.53 & 0.26 & 0.44 & 0.21 & 0.19 \\
\hline $\mathrm{Cd}$ & 0.05 & 0.06 & 0.03 & 0.04 & 0.04 \\
\hline $\mathrm{Co}$ & 0.36 & 0.45 & 0.27 & 0.19 & 0.52 \\
\hline $\mathrm{Cr}$ & 1.96 & 1.97 & 2.62 & 1.03 & 3.37 \\
\hline $\mathrm{Mn}$ & 1.94 & 0.87 & 1.38 & 0.96 & 2.75 \\
\hline $\mathrm{Zn}$ & 4.10 & 3.83 & 5.87 & 4.76 & 2.91 \\
\hline $\mathrm{Pb}$ & 1.06 & 2.86 & 0.86 & 2.07 & 2.36 \\
\hline $\mathrm{Sb}$ & 0.08 & 0.07 & 0.03 & 0.04 & 0.03 \\
\hline $\mathrm{Sn}$ & 0.27 & 0.72 & 0.48 & 0.64 & 0.23 \\
\hline $\mathrm{Te}$ & 0.04 & 0.03 & 0.02 & 0.03 & 0.04 \\
\hline $\mathrm{Fe}$ & 2.41 & 4.27 & 2.19 & 3.76 & 3.23 \\
\hline $\mathrm{Ni}$ & 5.36 & 3.25 & 2.67 & 5,23 & 3.86 \\
\hline $\mathrm{P}$ & 6.70 & 3.87 & 7.8 & 5.32 & 5,45 \\
\hline $\mathrm{Si}$ & 6.02 & 4.64 & 3.4 & 4.67 & 6.43 \\
\hline $\mathrm{O}_{2}$ & 7.00 & 8.00 & 12.00 & 9.00 & 10.00 \\
\hline \multicolumn{7}{c}{ Alloy additive content [wt.\%] } \\
\hline $\mathrm{Ag}$ & 2.92 & 3.96 & 4.93 & 6.03 & 7.06 \\
\hline $\mathrm{Cu}$ & rest & rest & rest & rest & rest \\
\hline \multicolumn{5}{c}{}
\end{tabular}


The density of the obtained alloys was determined using the classic method of measuring the mass of the samples in water and in air, with the knowledge of the liquid and air density at the temperature of the measurement. The influence of the silver content on the density of the obtained alloy is presented in Figure 1. As shown by the obtained results, the dependence of the silver content in the alloy and its density is quasi-linear.

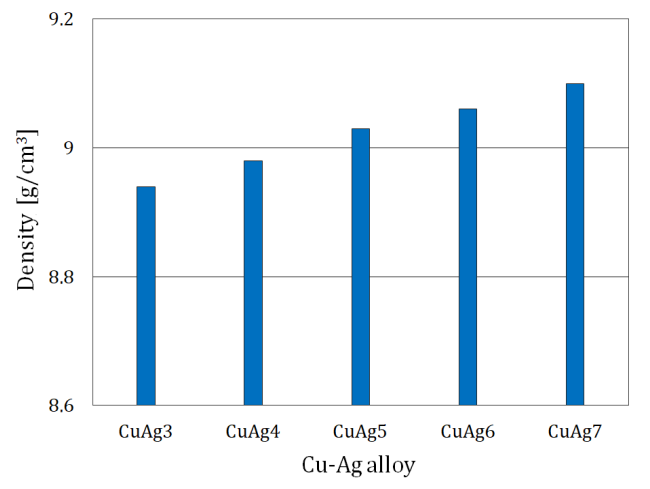

Fig. 1. The influence of the silver content on the density of the alloy

Figure 2 shows the Energy Dispersive X-Ray Analysis (EDX) of the element distribution on the example of CuAg6 alloy, which clearly shows areas rich in silver and copper formed during crystallization process carried out faster than equilibrium speed.
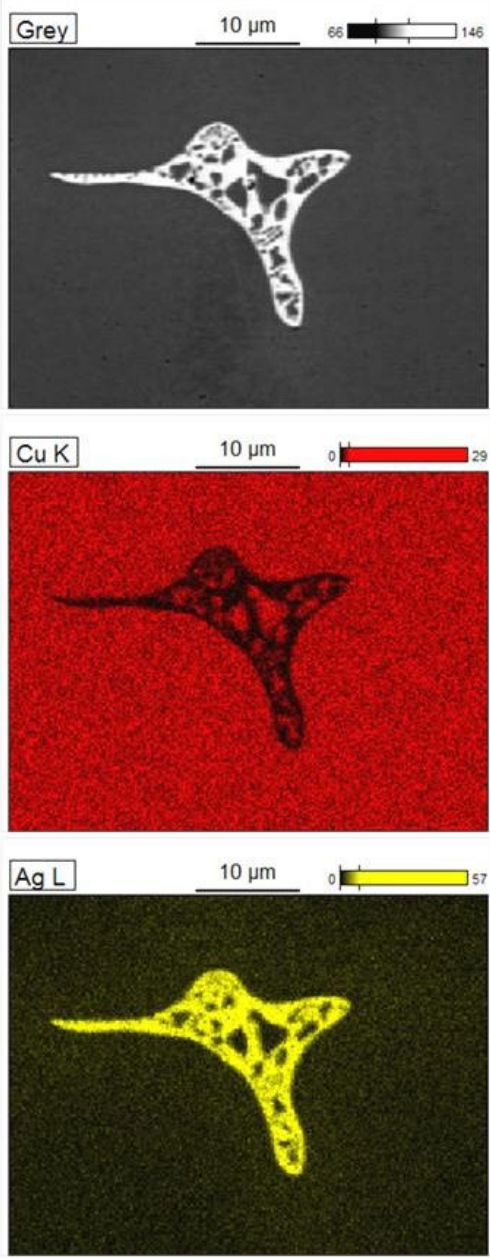

Fig. 2. EDX microanalysis of $\mathrm{Cu}$ and $\mathrm{Ag}$ distribution on the example of CuAg6 alloy longitudinal section
Figure 3 presents images of the obtained alloys obtained using Scanning Electron Microscope (SEM). The microstructure of the material in the as-cast state exhibits precipitates, areas rich in silver (white) which separated from the alloy matrix consisting mainly of copper (grey). The precipitates of silver may be observed due to the accelerated, non-equilibrium crystallization process.

a)

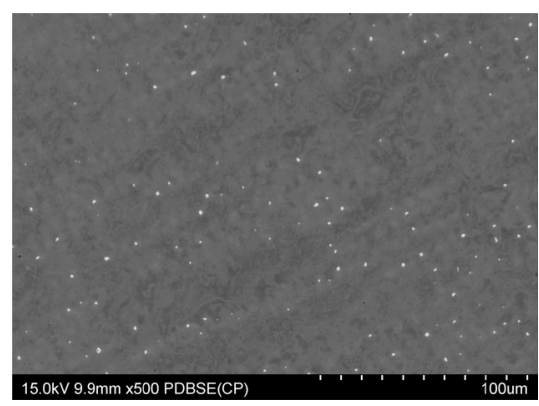

b)

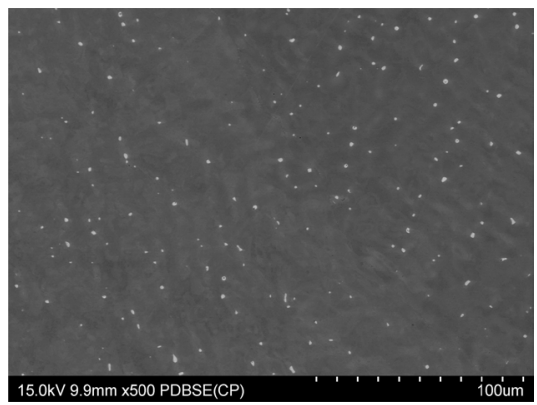

c)

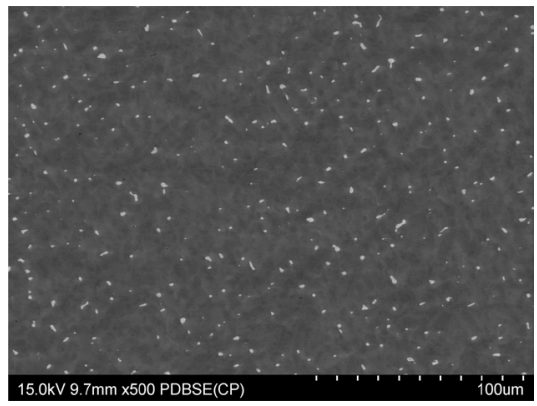

d)

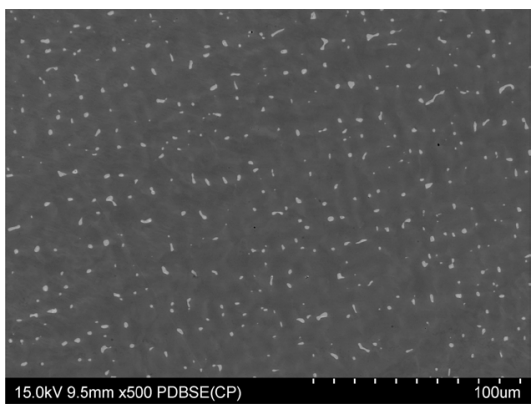

e)

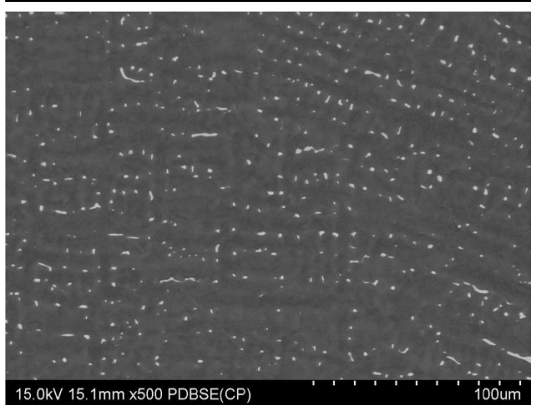

Fig. 3. SEM analysis of the longitudinal section of $\mathrm{Cu}-\mathrm{Ag}$ cast rods with various silver contents: a) CuAg3; b) CuAg4; c) CuAg5; d) CuAg6; e) $\mathrm{CuAg} 7$ 
Figures 4 and 5 presents the effect of silver addition to copper on the mechanical properties of $\mathrm{Cu}-\mathrm{Ag}$ alloys in the as-cast state with silver content ranging between 3 and $7 \mathrm{wt} . \%$ and Figure 6 presents the influence of the addition of silver to copper on the electrical conductivity of the tested alloys again in the as-cast state.

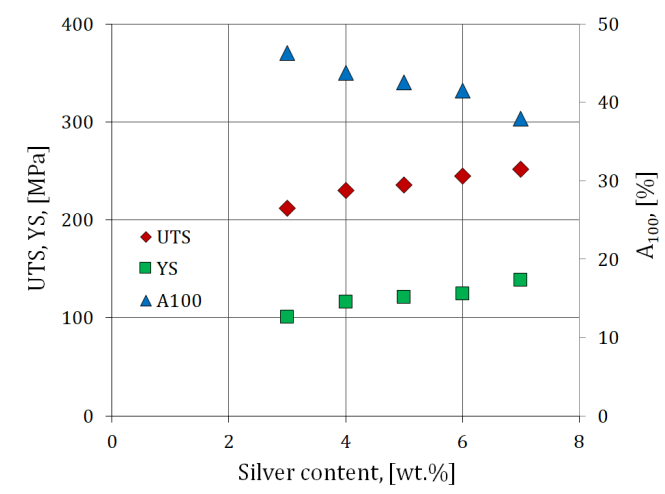

Fig. 4. The influence of the silver content on the mechanical properties of $\mathrm{Cu}-\mathrm{Ag}$ alloys with silver content ranging between 3 and $7 \mathrm{wt} . \%$

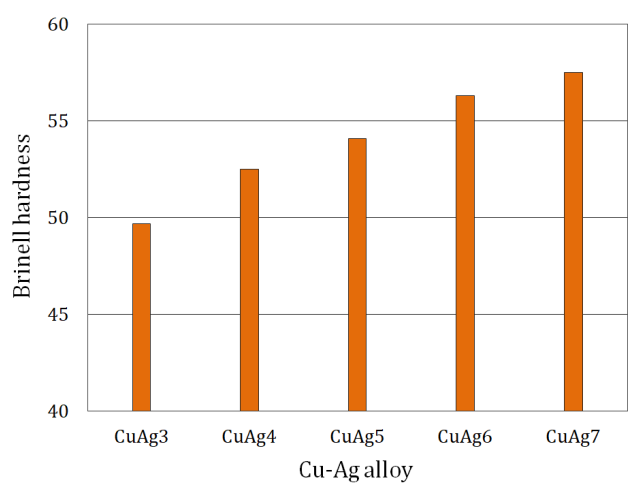

Fig. 5. The influence of the silver content on the hardness of $\mathrm{Cu}-\mathrm{Ag}$ alloys, with silver content ranging between 3 and 7 wt.\%

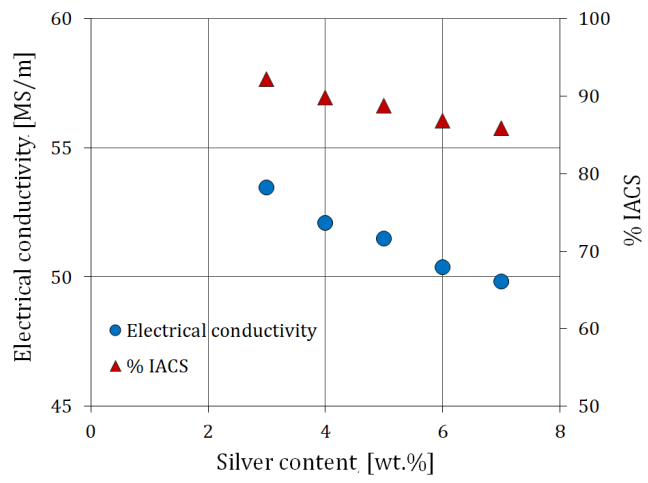

Fig. 6. The influence of the silver content on the electrical conductivity of $\mathrm{Cu}-\mathrm{Ag}$ alloys, with silver content ranging between 3 and $7 \mathrm{wt} . \%$

In order to correctly convert the resistance of the tested alloys to the standardized temperature of $20^{\circ} \mathrm{C}$, their temperature coefficients of resistance were determined. $\mathrm{Cu}-\mathrm{Ag}$ alloys in the as-cast state were drawn with the total true strain of 2.75 to the diameter of $2.4 \mathrm{~mm}$. Such wires were placed in the measuring rail of the Thomson bridge and placed in the heating chamber with the temperature range of $20^{\circ} \mathrm{C}$ and $120^{\circ} \mathrm{C}$. After the thermal state of the wires was stabilized, their electrical resistance was measured. Based on the obtained data, the values of the temperature coefficients of resistance were calculated for each alloy in order to depict the temperature influence on the dynamics of electrical resistance changes (Fig. 7). Among the tested range $\left(20-120^{\circ} \mathrm{C}\right)$ and silver alloy additive (3-7 wt.\% of $\mathrm{Ag}$ ) for wires drawn from materials in the as-cast state, the changes in the values of temperature coefficients of resistance are quasi-linear.

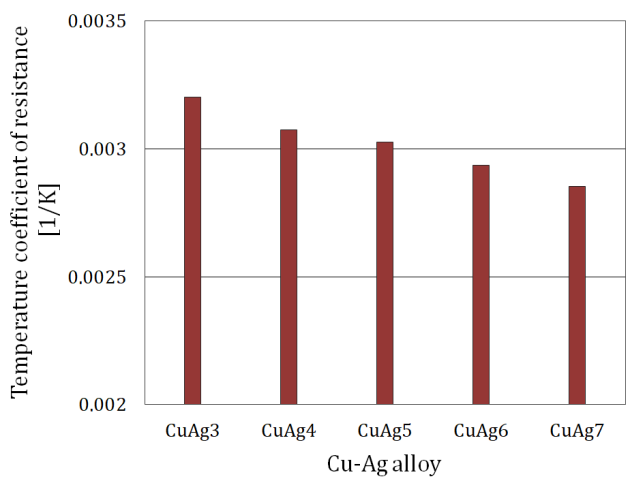

Fig. 7. The influence of the silver content on the value of temperature coefficients of resistance of $\mathrm{Cu}-\mathrm{Ag}$ alloys after wire drawing process of material in the as-cast state

Figures 8 and 9 present the effect of the amount of deformation on the mechanical and electrical properties. It may be stated that the alloy deformation with the true strain exceeding 6 allows a tensile strength ranging between 800 and $1000 \mathrm{MPa}$ to be obtained, however, it results in a significant decrease in the electrical conductivity of the tested materials.

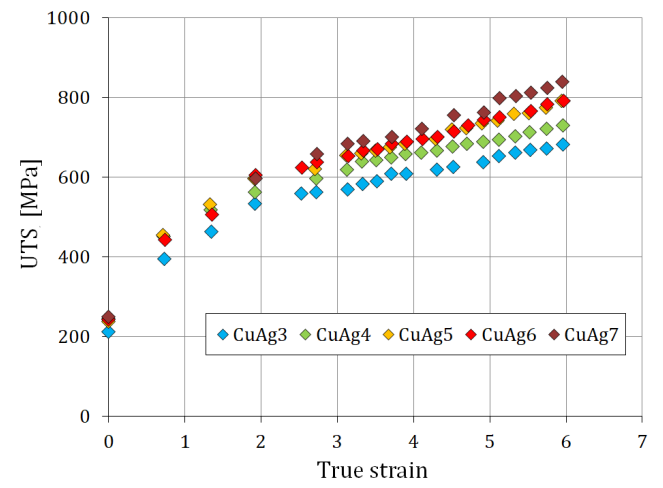

Fig. 8. The evolution of the ultimate tensile strength (UTS) in terms of the amount of deformation applied in the wire drawing process of the material in the as-cast state

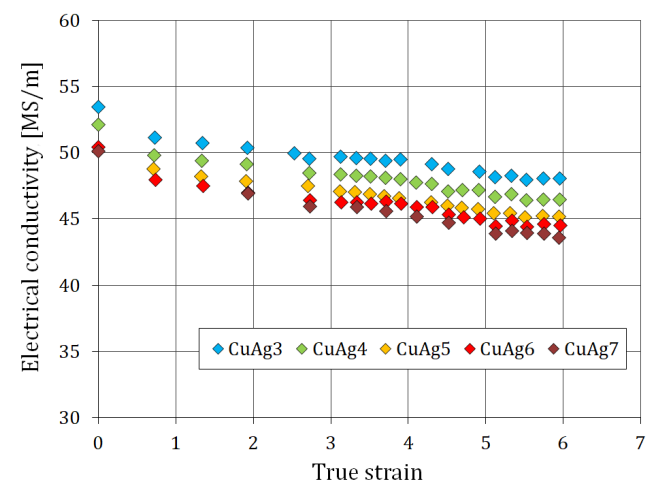

Fig. 9. The evolution of the electrical conductivity in terms of the amount of deformation applied in the wire drawing process of the material in the as-cast state 
Considering the course of the work-hardening curves, a high strengthening of the materials may be observed in the wire drawing process. Applying a true strain of 6 in the drawing process resulted, depending on the alloy, with 3-4 fold increase in Ultimate Tensile Strength (UTS). The course of the work-hardening curves is also specific. From a certain amount of deformation, the angle of the curve has a constant value suggesting the possibility of further intensive strengthening of these materials. However, as the mechanical properties significantly increase, the electrical conductivity of the drawn materials decreases. The more than $10 \%$ decrease in the electrical conductivity of the $\mathrm{Cu}-\mathrm{Ag}$ alloys may be explained by the particularly intensive generation of structural defects, disturbances of the crystal lattice, or the multiplication of dislocation accumulations throughout the plastic deformation of materials. Increasing the set of mechanical and electrical properties may be prospectively achieved by applying a prior to metal working, suitable heat treatment of the alloys in the as-cast state. Applying supersaturation and aging processes in order to introduce silver into the solution and then its precipitation as much as possible in the form of numerous fine particles may contribute to the intensification of the alloy strengthening mechanisms. Purifying the copper matrix of silver by its precipitation may at the same time contribute to increasing the alloy's electrical conductivity.

\section{CONCLUSIONS}

Preliminary research results were presented in this paper on the technology development for the manufacturing process of $\mathrm{Cu}$-Ag wires which may be used for the construction of electromagnet coils generating a strong impulse magnetic field. Due to the specific design of such coils, the knowledge of the distribution of not only the magnetic field but also Lorenz forces at the coils cross-section is crucial. It is significant to choose the shape, size of the cross-section of the wires and their mechanical and electrical properties. It is possible to further optimize the construction of the coil using wires with various mechanical and electrical properties at the cross-section of the coil windings. Therefore, it is possible to use various $\mathrm{Cu}-\mathrm{Ag}$ alloys for the construction of the coil. This may contribute to generating the maximum value of the magnetic field for a given coil structure and at the same time extending its operating time.

\section{REFERENCES}

[1] Freudenberger J., Klauss H.-J., Heinze K., Gaganov A., Schaper M. \& Schultz L. (2008). Fatigue of highly strengthened Cu-Ag alloys. International Journal of Fatigue, 30 (3), 437-443. Doi: https:// doi.org/10.1016/J.JJFATIGUE.2007.04.009.

[2] Van Bockstal L., Li L., Harrison N., Heremans G., Herlach F, Starett B. \& Clark G.G. (1996). Long life pulsed high field magnets with CuAg conductor and internal reinforcement. IEEE, 32(4), 2514-2417. Doi: https://doi.org/10.1109/20.511384.

[3] Wood J.T., Embury J.D. \& Ashby M.F. (1997). An approach to materials processing and selection for high-field magnet design. Acta Materialia, 45(3), 1099-1104. Doi: https://doi.org/10.1016/ S1359-6454(96)00220-0.

[4] Strzępek P., Mamala A., Zasadzińska M., Noga P. \&Sadzikowski M. (2020). The influence of the continuous casting conditions on the properties of high-strength two-phase CuMg alloys. Materials, 13(21), 4805. Doi: https://doi.org/10.3390/ma13214805.
[5] Strzępek P., Mamala A., Zasadzińska M., Franczak K. \& Jurkiewicz B. (2019). Research on the drawing process of $\mathrm{Cu}$ and $\mathrm{CuZn}$ wires obtained in the cryogenic conditions. Cryogenics, 100,11-17. Doi: https://doi.org/10.1016/j.cryogenics.2019.03.007.

[6] Zasadzińska M., Knych T., Smyrak B. \& Strzępek P. (2020). Investigation of the dendritic structure influence on the electrical and mechanical properties diversification of the continuously casted copper strand. Materials, 13(23), 5513. Doi: https://doi.org/ 10.3390/ma13235513.

[7] Zasadzińska M. \& Knych T. (2019). The morphology of eutectic copper oxides $\mathrm{I}\left(\mathrm{Cu}_{2} \mathrm{O}\right)$ in the processing of wire rod and wires made from ETP grade copper. Archives of Metallurgy and Materials, 64, 1611-1616. Doi: https://doi.org/10.24425/amm.2019.130134.

[8] Zasadzińska M., Knych T., Strzępek P., Jurkiewicz B. \& Franczak K. (2019). Analysis of the strengthening and recrystallization of electrolytic copper (Cu-ETP) and oxygen free copper (Cu-OF). $A r$ chives of Civil and Mechanical Engineering, 19(1), 186-193. Doi: https://doi.org/10.1016/j.acme.2018.09.008.

[9] Han K., Embury J.D., Sims J.R., Campbell L.J., Schneider-Muntau H.-J., Pantsyrnyi V.I., Shikov A., Nikulin A. \& Vorobieva A. (1999). The fabrication, properties and microstructure of $\mathrm{Cu}-\mathrm{Ag}$ and $\mathrm{Cu}-\mathrm{Nb}$ composite conductors. Material Science and Engineering: A, 267(1), 99-114. Doi: https://doi.org/10.1016/S0921-5093(99)00025-8.

[10] Zasadzińska M., Strzępek P., Mamala A. \& Noga P. (2020). Reinforcement of Aluminium-Matrix Composites with Glass Fibre by Metallurgical Synthesis. Materials, 13(23), 5441. Doi: https:// doi.org/10.3390/ma13235441.

[11] Sakai Y., Inoue K., Asano T., Wada H. \& Maeda H. (1991). Development of high-strength high-conductivity $\mathrm{Cu}-\mathrm{Ag}$ alloys for high-field pulsed magnet use. Applied Physics Letters, 59(23), 2965-2967. Doi: https://doi.org/10.1063/1.105813.

[12] Hirota T., Imai A., Kumano T., Ichihara M., Sakai Y., Inoue K. \& Maeda H. (1994). Development of Cu-Ag alloys conductor for high field magnet. IEEE Transaction on Magnetics, 30(4), 1891-1894. Doi: https://doi.org/10.1109/20.305631.

[13] Sakai Y. \& Schneider-Muntau H.J. (1997). Ultra-high strength, high conductivity $\mathrm{Cu}-\mathrm{Ag}$ alloy wires. Acta Materialia, 45(3), 1017-1023. Doi: https://doi.org/10.1016/S1359-6454(96)00248-0.

[14] Sakai Y., Inoue K. \& Maeda H. (1995). New high-strength, high-conductivity $\mathrm{Cu}-\mathrm{Ag}$ alloy sheets. Acta Metallurgica et Materialia, 43(4),1517-1522. Doi: https://doi.org/10.1016/0956-7151(94)00376-S.

[15] Lin J. \& Meng L. (2008). Effect of aging treatment on microstructure and mechanical properties of $\mathrm{Cu}-\mathrm{Ag}$ alloys. Journal of Alloys and Compounds, 454(1-2), 150-155. Doi: https://doi.org/ 10.1016/J.JALLCOM.2006.12.073.

[16] Gupta S.P. (1998). Kinetics of discontinuous precipitation and dissolution in Cu-Ag alloys. Canadian Metallurgical Quarterly, 37(2), 141-159. Doi: https://doi.org/10.1179/cmq.1998.37.2.141.

[17] Bao G., Xu Y., Huang L., Lu X., Zhang L., Fang Y., Meng L. \& Liu J. (2016). Strengthening effect of Ag precipitates in Cu-Ag alloys: A quantitative approach. Materials Research Letters, 4(1), 37-42. Doi: https://doi.org/10.1080/21663831.2015.1091795.

[18] Cho H., Lee B.S., Kang B.H. \& Kim K.Y. (2008). Ageing behavior of Cu-Ag alloys. Advanced Materials Research, 47-50, 1051-1054. Doi: https://doi.org/10.4028/www.scientific.net/AMR.47-50.1051.

[19] Meng L. \& Liu J.B. (2007). Progress and currentstatusin research on nanostructured $\mathrm{Cu}-\mathrm{Ag}$ microcomposites for conductor wires. $\mathrm{Ma}$ terial Science Forum, 539-543, 2798-2803. Doi: https://doi.org/ 10.4028/www.scientific.net/MSF.539-543.2798.

[20] Gaganov A., Freudenberger J., Grunberger W. \& Schultz L. (2004). Microstructural evolution and its effect on the mechanical properties of $\mathrm{Cu}-\mathrm{Ag}$ microcomposites. Zeitschrift für Metallkunde, 95(6), 425-432.

[21] Ning Y., Zhang X. \& Wu Y. (2007). Electrical Conductivity of Cu-Ag in situ filamentary composites. Transactions of Nonferrous Metals Society of China, 17(2), 378-383. Doi: https://doi.org/10.1016/ S1003-6326(07)60102-2.

[22] Strzępek P., Mamala A., Zasadzińska M., Kiesiewicz G. \& Knych T.A. (2021). Shape analysis of the elastic deformation region throughout the axi-symmetric wire drawing process of ETP grade copper. Materials, 14(16), 4713. Doi: https://doi.org/10.3390/ ma14164713. 